\title{
Toll-like receptor 2 controls expansion and function of regulatory $T$ cells
}

\author{
Roger P.M. Sutmuller, ${ }^{1}$ Martijn H.M.G.M. den Brok, ${ }^{1}$ Matthijs Kramer, ${ }^{1}$ Erik J. Bennink, ${ }^{1}$ \\ Liza W.J. Toonen, ${ }^{1}$ Bart-Jan Kullberg, ${ }^{2}$ Leo A. Joosten, ${ }^{3}$ Shizuo Akira, ${ }^{4}$ \\ Mihai G. Netea, ${ }^{2}$ and Gosse J. Adema ${ }^{1}$
}

\begin{abstract}
${ }^{1}$ Department of Tumor Immunology, ${ }^{2}$ Department of Internal Medicine, and ${ }^{3}$ Department of Rheumatology, Nijmegen Centre for Molecular Life Sciences, Radboud University Nijmegen Medical Centre, Nijmegen, The Netherlands. ${ }^{4}$ Department of Host Defense, Research Institute for Microbial Diseases, Osaka University, Osaka, Japan.
\end{abstract}

\begin{abstract}
Tregs play a central role in the suppression of immune reactions and prevention of autoimmune responses harmful to the host. During acute infection, however, Tregs might hinder effector $T$ cell activity directed toward the elimination of the pathogenic challenge. Pathogen recognition receptors from the TLR family expressed by innate immune cells are crucial for the generation of effective immunity. We have recently shown the $\mathrm{CD}^{+} \mathrm{CD}^{+} 5^{+}$Treg subset in $T L R 2^{-/-}$mice to be significantly reduced in number compared with WT littermate control mice, indicating a link between Tregs and TLR2. Here, we report that the TLR2 ligand Pam ${ }_{3} \mathrm{Cys}_{\text {, }}$ but not LPS (TLR4) or CPG (TLR9), directly acts on purified Tregs in a MyD88-dependent fashion. Moreover, when combined with TCR stimulation, TLR2 triggering augmented Treg proliferation in vitro and in vivo and resulted in a temporal loss of the suppressive Treg phenotype in vitro by directly affecting Tregs. Importantly, WT Tregs adoptively transferred into $T L R 2^{-/-}$mice were neutralized by systemic administration of TLR2 ligand during the acute phase of a Candida albicans infection, resulting in a 100-fold reduced C. albicans outgrowth. This demonstrates that in vivo TLR2 also controls the function of Tregs and establishes a direct link between TLRs and the control of immune responses through Tregs.
\end{abstract}

\section{Introduction}

It is now evident that the rediscovered cells known as Tregs play a dominant role in our immune systems (reviewed in refs. 1,2). Two types of Tregs can be distinguished: $\mathrm{CD} 4^{+} \mathrm{CD} 25^{+}$intrinsic Tregs, which are already present in the naive individual, and those induced in the periphery. TCR triggering renders intrinsic Tregs able to suppress both $\mathrm{CD}^{+}$and $\mathrm{CD}^{+}$lymphocytes $(3,4)$ via a process that is cell-cell-contact dependent, though the exact mechanism is not yet identified. Several studies have shown that their depletion results in autoimmune syndromes such as thyroiditis, gastritis, insulindependent diabetes mellitus, colitis, and arthritis (5-8). On the other hand, active Tregs hinder the induction of immune responses against pathogens and tumors (9-11), emphasizing the importance of a tight control of these regulators. Importantly, IL-2 (3), IL-6 (12), and strength of the TCR signal (13) have been reported to release effector $\mathrm{T}$ cells from Treg-mediated suppression.

Besides these control mechanisms regulating the sensitivity of effector $\mathrm{T}$ cells to suppression, still many questions remain regarding regulatory mechanisms acting at the level of Tregs. Tregs are able to proliferate in normal unmanipulated mice (14). In addition, the finding that CD80/CD86-deficient mice have significantly decreased numbers of Tregs indicates a role for costimulation in Treg homeostasis (15). Furthermore, mature DCs have been reported to induce Treg expansion (16). In line with this, TLR-triggered DCs were recently shown to induce Treg proliferation by a

Nonstandard abbreviations used: CTLA-4, cytotoxic T lymphocyte-associated antigen-4; GITR, glucocorticoid-induced TNF receptor family-related protein; MALP-2, macrophage-activating lipopeptide-2; MFI, mean fluorescence intensity; OVA-peptide, OVA-derived peptide; PAM, Pam ${ }_{3}$ Cys-SKKKK; PAMP, pathogen-associated molecular pattern; PBGD, porphobilinogen deaminase.

Conflict of interest: The authors have declared that no conflict of interest exists. Citation for this article: J. Clin. Invest. 116:485-494 (2006). doi:10.1172/JCI25439. cooperative action of IL-1 and IL-6 (17). Together, these reports provide accumulating evidence that Treg anergy can be overcome when the right stimulatory environment is provided, showing that Tregs play a dynamic role in the control of immune responses.

One family of receptors involved in immune regulation is the TLR family, a class of receptors that recognizes pathogen-associated molecular patterns (PAMPs) or endogenous inflammationassociated molecules (18). So far, 13 receptors have been identified with different or overlapping ligands. TLR1/2 heterodimers, for example, can recognize bacterial lipoproteins, TLR4 recognizes LPS from Gram-negative bacteria, and TLR9 recognizes bacterial DNA (unmethylated CG motifs). TLRs are abundantly expressed on innate immune cells, such as macrophages and DCs, and TLR9induced IL- 6 production by DCs releases effector T cells from Treg-mediated suppression (12). Recently, TLRs were also found on T cells, including Tregs (19). Therefore, we hypothesized that some TLR ligands might directly affect Tregs. We have previously shown that Treg numbers in the circulation of TLR2 $2^{-/-}$mice, but not $T L R 4^{--}$mice, are significantly reduced compared with their WT littermate controls (10). Moreover, we and others have shown that the immune response against the fungal pathogen Candida albicans is affected by Tregs $(9,10)$. Using highly purified intrinsic Tregs and conventional $\mathrm{CD}^{+}{ }^{+} \mathrm{T}$ cells, we now show that TLR2 triggering on the intrinsic Tregs results in proliferation of these Tregs in vitro and in vivo. Moreover, in the presence of the TLR2 ligand $\mathrm{Pam}_{3} \mathrm{Cys}$, the suppressive phenotype of Tregs is temporarily abrogated in vitro and in vivo, thereby enhancing the antifungal response in an acute infection model. After removal of the TLR2trigger, Tregs fully regained their suppressive capabilities. These findings demonstrate that, in addition to their effects on APCs, TLR ligands can directly act on intrinsic Tregs, resulting in dynamic modulation of the immune response. 

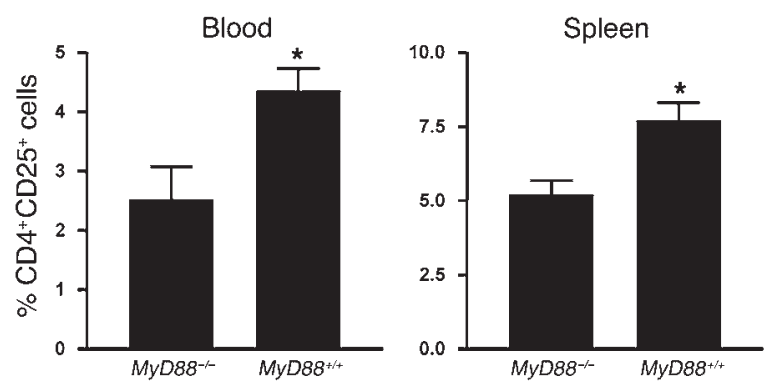

Figure 1

Decreased CD4 ${ }^{+} \mathrm{CD} 25^{+} \mathrm{T}$ cell numbers in MyD88-deficient mice. Blood and spleens from MyD88 $8^{-/-}$mice and their littermate $M y D 88^{+/+}$controls (4 per group) were analyzed by flow cytometry for relative $C D 4^{+} C D 25^{+}$ $T$ cell numbers. Data indicate mean percentage of $C D 4+C D 25^{+} T$ cell numbers of total CD4+ $\mathrm{T}$ cells \pm SEM. Representative results of 3 experiments are shown. ${ }^{*} P<0.02$ with WT controls.

\section{Results}

TLR2 signaling modulates $\mathrm{CD} 4^{+} \mathrm{CD} 25^{+} \mathrm{T}$ cell levels in vivo. Recently, we demonstrated decreased numbers of circulating $\mathrm{CD} 4^{+} \mathrm{CD} 25^{+}$Tregs in blood of TLR2 $2^{-/-}$mice, but not of TLR $4^{-/-}$mice (10). These findings suggest a role for TLR2 signaling in Treg homeostasis and/or function. As TLR2 signaling is critically dependent on the adaptor molecule MyD88 (18), we now determined the relative number of Tregs present in blood and spleen of MyD88-deficient mice and their WT littermate controls. As shown in Figure 1, MyD $88^{-/-}$mice, like TLR2 $2^{--}$mice (10), contained significantly lower numbers of $\mathrm{CD} 4^{+} \mathrm{CD} 25^{+} \mathrm{T}$ cells compared with their WT controls. In contrast, the percentage of $\mathrm{CD} 4^{+} \mathrm{CD} 25^{-}$conventional Th cells did not differ between MyD88 and control mice $(15.9 \pm 1.2$ and $17.6 \pm 1.5$, respectively) as well as TLR2 ${ }^{-/-}$mice and their controls $(17.7 \pm 2.1$ and $18.3 \pm 1.9$, respectively). In Supplemental Figure 1 (supplemental material available online with this article; doi:10.1172/
JCI25439DS1), representative $\mathrm{CD} 4^{+} \mathrm{CD} 25^{+} \mathrm{T}$ cell stainings from individual TLR2 $2^{--}$and $M y D 88^{--}$mice and their WT littermate controls are shown. The decreased Treg numbers in both the TLR2- and MyD88-KO mice indicate that a lack of TLR2 signaling is responsible for the observed decrease of $\mathrm{CD} 4^{+} \mathrm{CD} 25^{+} \mathrm{T}$ cell numbers in vivo.

These results thus demonstrate a relation between the TLR2/ MyD88 signaling pathway and Treg numbers in vivo.

TLR triggering in the presence of APCs modulates Tregs in vitro. TLR2 is expressed by cells of the innate immune system, including APCs, as well as by Tregs (19). TLR-triggering compounds are known to promote APC activation (18), resulting in the production of cytokines affecting $T$ cell function (12). Alternatively, some TLR ligands might directly act on Tregs. To address the role of TLRs in Treg function, we analyzed the effect of different TLR ligands on Treg proliferation in vitro. As expected, Tregs cultured in the presence of irradiated unstimulated APCs and soluble anti-CD3 did not display proliferation (Figure 2A), in accordance with their anergic state (20). Addition of various TLR ligands to these APC/Treg cocultures significantly enhanced their proliferative capacity, resulting in increased numbers of Tregs (Figure 2A). This observation is in line with a recent report describing inflammatory cytokine production by TLRtriggered DCs as resulting in increased proliferation of Tregs (17). The effect of TLR ligands on Treg proliferation is also reflected by the upregulation of the $T$ cell activation marker CD25. As shown in Figure 2B, addition of TLR ligands LPS (TLR4), $\mathrm{Pam}_{3} \mathrm{Cys}$-SKKKK (PAM, TLR2), or CpG (TLR9) all resulted in a significant increase in CD25 expression on Tregs. However, the increase in CD25 expression was most pronounced upon addition of PAM. Thus, these data show that in the presence of APCs, TLR ligands induce Treg proliferation and CD25 upregulation.

TLR2 triggering in the absence of APCs modulates Tregs in vitro. To investigate the direct effects of these TLR ligands on intrinsic Tregs and conventional CD $4^{+} \mathrm{CD} 25^{-}$Th cells, highly pure (>98\%) Tregs and Th cells were incubated with the TLR ligands plus anti-
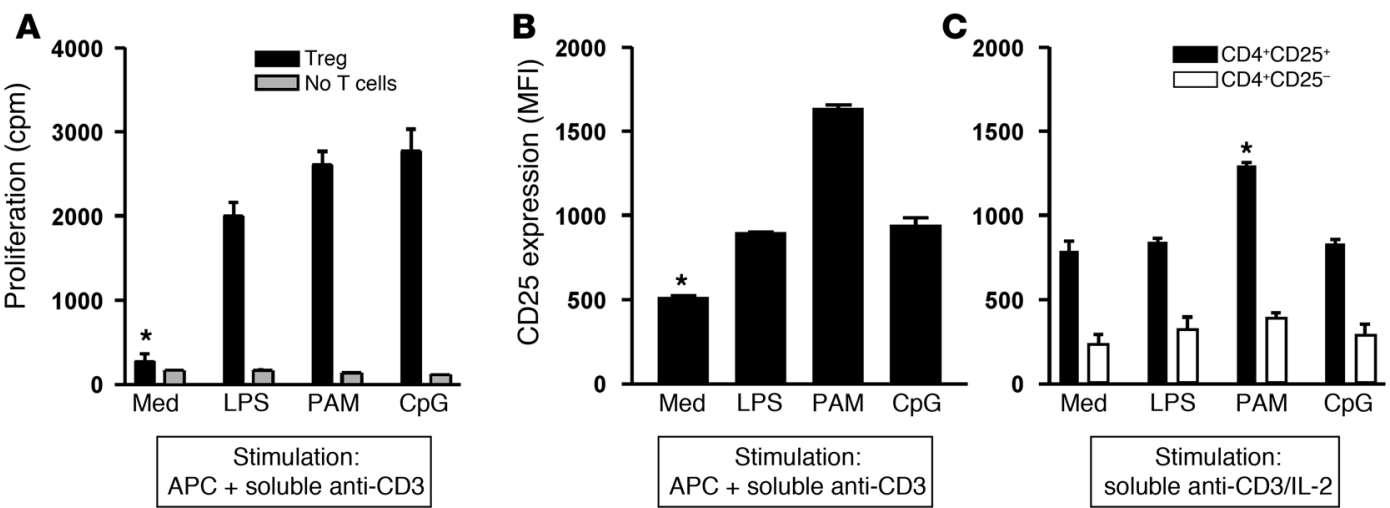

Figure 2

In vitro TLR2 signaling results in Treg proliferation. Below each graph, the specific T cell stimulation is indicated. (A) Proliferation of Tregs in the presence of irradiated APCs, anti-CD3, and TLR ligands. Irradiated APCs and anti-CD3 (medium control [med]) were cultured for 3 days with or without $10^{4}$ purified Tregs and with or without the addition of TLR ligands: purified LPS (TLR4), PAM (TLR2), or CpG (TLR9). Values indicate average counts per minute of triplicate wells \pm SD. ${ }^{*} P<0.02$ for medium control compared with TLR ligands. (B and C) CD25 expression by purified $\mathrm{CD} 4{ }^{+} \mathrm{CD} 25^{+/-}$T cells. (B) Tregs were cultured for 3 days in the presence of irradiated APCs and anti-CD3 (medium control) or with addition of purified LPS, PAM, or CpG. CD25 expression was measured by flow cytometry. Values indicate average MFI of anti-CD25-FITC-stained $\mathrm{CD} 4{ }^{+}$cells of triplicates $\pm \mathrm{SD}$. ${ }^{*} P<0.02$ for medium control compared with TLR ligands. (C) $T$ cell activation in the absence of APCs. Purified CD4+CD25+ Tregs or CD4+CD25- conventional Th cells were cultured with IL-2 and soluble anti-CD3 (medium control) or with the addition of purified LPS, PAM, or CpG (no APCs present). After 3 days, CD25 expression was measured by flow cytometry. Values indicate average MFI of triplicates $\pm S D$. One representative experiment out of 3 is shown. ${ }^{*} P<0.02$ PAM with medium control. 

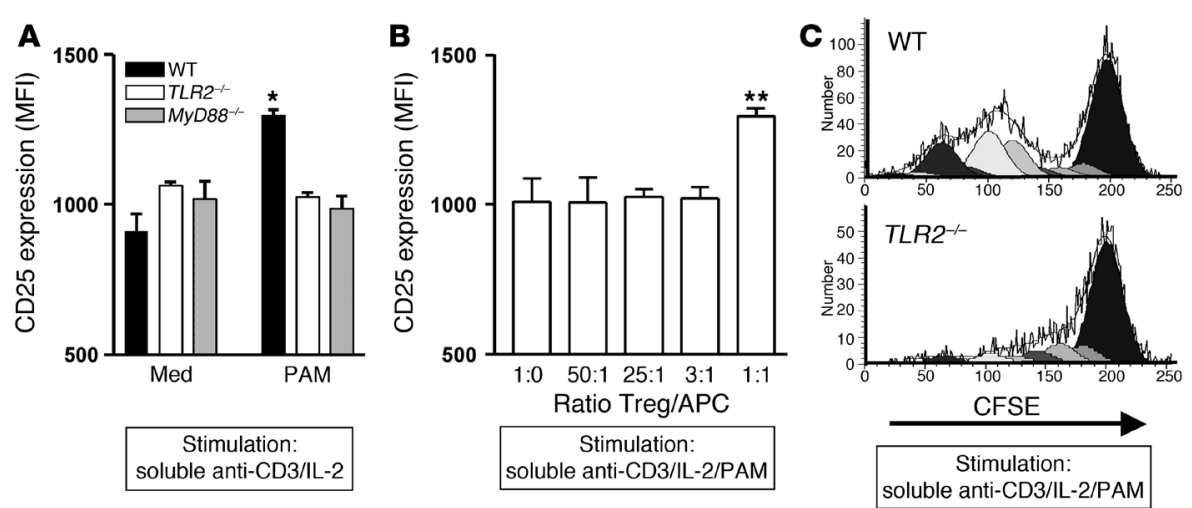

\section{Figure 3}

PAM induces CD25 expression through TLR2 signaling. Below each graph, the specific T cell stimulation is indicated. (A) TLR2 and MyD88 expression is required for PAM-mediated increase of CD25 expression. Purified WT, TLR2 ${ }^{-1-}$, and MyD88 $8^{-/}$CD4 $4^{+}$CD25 $5^{+}$T cells were cultured for 3 days with anti-CD3, IL-2 (medium control), or with the addition of PAM. Subsequently, the cells were harvested and CD25 expression was analyzed by flow cytometry. Values indicate average MFI from triplicate wells \pm SD. ${ }^{*} P<0.02$ with medium control. A representative result of 3 experiments is shown. (B) High numbers of WT APCs are required to increase CD25 expression on $T L R 2^{-/-}$Tregs. Purified CD4+CD25+ T cells from TLR2 ${ }^{-1-}$ mice were incubated for 3 days with increasing amounts of WT APCs plus the TLR2 ligand PAM and anti-CD3, and subsequently, CD25 expression was analyzed by flow cytometry. Values indicate average MFI from triplicates \pm SD. ${ }^{* *} P<0.05$ with medium control. (C) Proliferation of CFSE-labeled freshly isolated WT and TLR2--- Tregs after stimulation with soluble anti-CD3, IL-2, and PAM. After 4 days, proliferation resulting in a decrease of fluorescent signal in the daughter cells was analyzed by flow cytometry and ModFit analysis software. Representative results of 2 experiments are shown.

CD3 antibodies and IL-2 but, importantly, in the absence of APCs. Interestingly, only the addition of PAM, but not purified LPS or $\mathrm{CpG}$, resulted in profoundly increased expression of $\mathrm{T}$ cell activation markers CD25 (Figure 2C and Supplemental Figure 2A) and CD69 (not shown) on Tregs. Only limited effects of TLR stimulation were observed for conventional Th cells.

In contrast to highly purified LPS (TLR4 ligand), the synthetic TLR2 ligands Pam ${ }_{3}$ Cys (TLR1/2) and macrophage-activating lipopeptide-2 (MALP-2; TLR2/6) as well as the natural TLR2 ligands in peptidoglycan, commercial (nonpure) LPS, and heat-killed C. albicans (all containing TLR2 ligands) induced CD25 upregulation, indicating that besides synthetic TLR2 ligands, natural TLR2 ligands also directly affect Tregs (Supplemental Figure 2B).

Of note, differences between the basal levels of CD25 expression of Tregs stimulated with either anti-CD3/APCs or anti-CD3/ IL-2 (Figure 2, B and C) can be explained by the different amounts of (co)stimulatory signals Tregs receive with each different stimulation approach. Both approaches use anti-CD3 but differ in the use of APCs versus IL-2. Yet, in the absence of APCs, the TLR2 ligand PAM results in increased expression of $T$ cell activation markers on Tregs.

TLR2 signaling by Tregs induces expression of CD25. To exclude that the effects caused by PAM were the result of contamination in the synthetic PAM preparation, we tested Tregs purified from TLR2- and MyD88-deficient mice. We found that only WT Tregs responded to PAM with an increase in CD25 expression, whereas no effect was observed for TLR2-deficient and MyD88-deficient Tregs (Figure 3A), indicating that PAM acts through both TLR2and MyD88-dependent signaling pathways.

To further exclude that a small amount of contaminating cells within the FACS-sorted Treg preparations are responsible for the observed effects, we used $\mathrm{CD} 4^{+} \mathrm{CD} 25^{+} \mathrm{T}$ cells from $T L R 2^{-/-}$mice that we found unable to respond to PAM (Figure 3A). To mimic a cellular contamination, increasing amounts of WT syngeneic APCs were added to FACS-sorted TLR2 $2^{--}$Tregs in the presence of
PAM/anti-CD3. The results (Figure 3B) showed that almost equal numbers of WT APCs were needed to increase CD25 expression on the TLR2-deficient Tregs. This shows that in our experiments with highly pure WT Tregs (Figures 2C and 3A), PAM must have acted directly on Tregs.

These data thus indicate that the TLR2 ligand PAM, but not TLR4 or TLR9 ligands, is able to directly trigger Tregs in a MyD88-dependent manner.

TLR2 signals induce Treg expansion in vitro. In an attempt to establish long-lived Treg cultures, TLR ligands were added to a culture of purified Treg feeder cells and supplemented with soluble antiCD3 and IL-2. In a primary stimulation, the addition of PAM, LPS, or CPG increased the proliferation of Tregs (Figure 2B). In multiple experiments, however, the addition of LPS or CPG was not sufficient to obtain viable Treg lines (not shown). In contrast, coculturing Tregs in the presence of PAM resulted repeatedly in the generation of a pure $\mathrm{CD} 4^{+} \mathrm{CD} 25^{+} \mathrm{T}$ cell line. Analysis of proliferation of CFSE-labeled freshly isolated WT- and TLR2-/-derived Tregs showed that WT but not TLR2-1- Tregs responded to stimulation with anti-CD3 and TLR2 ligand (Figure 3C). The phenotypic characteristics of these PAM-cultured Tregs were consistent with the reported intrinsic Treg markers, including CD4, CD25, cytotoxic T lymphocyte-associated antigen-4 (CTLA-4), glucocorticoid-induced TNF receptor family-related protein (GITR), CD103 (1, 21), and the Treg-specific transcription factor Foxp3 (22-24) (Figure 4). Of note, TLR2 $2^{-1-}$ Tregs that were treated in vitro with the same PAM-based expansion protocol did not proliferate (see Figure 3C) but did express similar amounts of CD4, CD25, CTLA-4, GITR, CD103, and Foxp3 (data not shown). Importantly, WT Tregs expressed low but significant amounts of TLR2 mRNA (Figure 4B) as well as protein (Figure 4C), which further strengthens our hypothesis of TLR2-mediated control of Treg function.

Cooperation between TLR2- and TCR signaling results in Treg expansion. To address the effects of combined TLR 2 and TCR signaling 
A
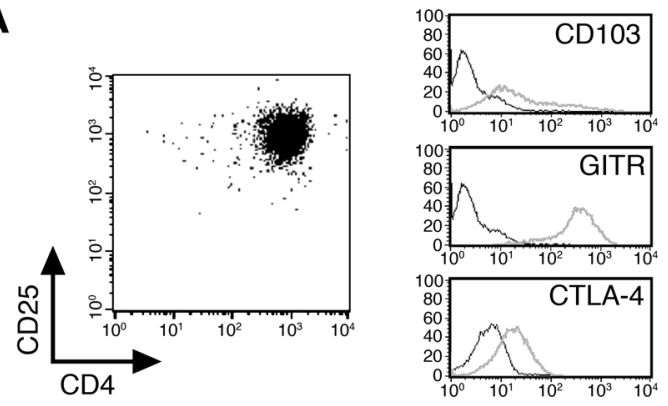

B

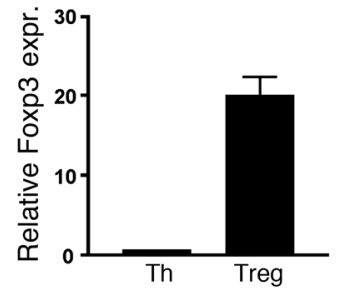

c
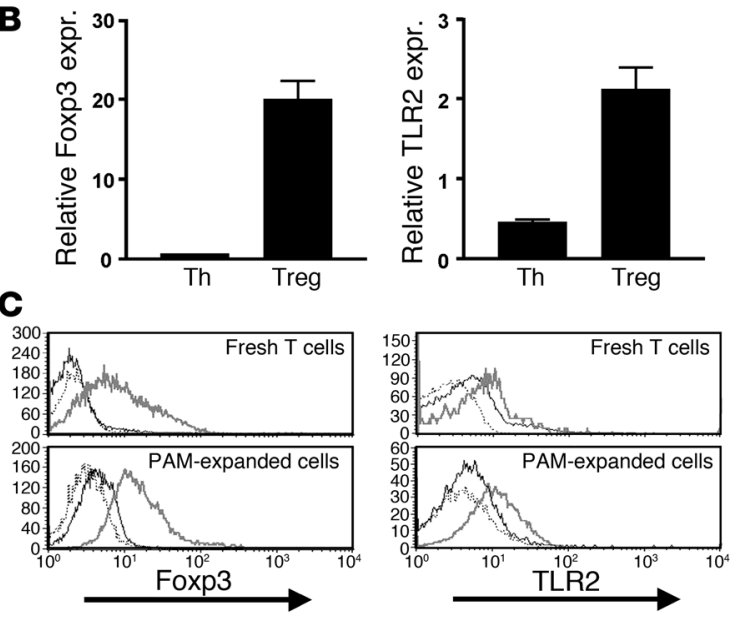

in more detail, we analyzed the expression oaf the activation marker CD25 on in vitro-expanded Tregs in time. Our results show that TLR2 triggering of Tregs cooperated with anti-CD3-mediated TCR stimulation, resulting in maximal increased CD25 expression (Figure 5A) as compared with either stimulation alone. This shows that these cells remained responsive toward TLR2 stimulation and that optimal Treg activation requires both TCR and TLR2 signaling. This is further demonstrated by the observation that the addition of PAM in combination with a strong TCR signal (applying plate-bound anti-CD3) induced proliferation of Tregs, in contrast to TLR4 or TLR9 ligands (Figure 5B). The proliferation of Tregs induced by TLR2 triggering and/or TCR stimulation was further visualized by their CFSE dilution profile. Illustrative for their anergic state, comparing untreated Treg (medium) with anti-CD3-stimulated Treg, TCR signaling alone did not induce proliferation in these cells (Figure 5C). However, the addition of TLR2 ligand alone induced some proliferation of Tregs as shown by the decrease in CFSE signal. However, maximal proliferation of Tregs was observed when both TCR and TLR2 stimulations were applied. Of note, although PAM increased the proliferation of Tregs up to 10 times, Treg proliferation remained low compared with the proliferation observed for conventional $\mathrm{T}$ cells.

TLR2-expanded Tregs remain suppressive. Importantly, to address whether the expanded Tregs were still capable of suppressing conventional $\mathrm{T}$ cell responses, we performed in vitro suppression assays. The PAM-expanded Tregs were rested for at least 5 days in the absence of PAM and subsequently cocultured with freshly isolated $\mathrm{CD}^{+} \mathrm{CD} 25^{-}$conventional T cells. Analysis of the T cell response after 3 days showed that Tregs efficiently suppressed proliferation (Figure 6A) as well as IFN- $\gamma$ production (Supplemental Figure 2C) of freshly isolated conventional T cells (Th). Conven-

\section{Figure 4}

Phenotype of PAM-expanded Treg. Expression of intrinsic Treg specific markers on PAM-expanded resting (7 days after stimulation with PAM) Tregs was analyzed by flow cytometry and quantitative PCR. (A) The PAM-expanded Tregs expressed the markers CD4, CD25, GITR, CTLA-4, and CD103 (indicated by thick gray, lines; corresponding isotype controls are indicated by thin, black lines). CTLA-4 was detected by standard intracellular staining procedure. (B) Expression (expr.) of Foxp3 (left panel) and TLR2 (right panel) mRNA by resting PAM-expanded Tregs and conventional CD25- T helper cells was determined by quantitative PCR. The quantitative PCR results are indicated as mean relative mRNA expression from 3 replicate measurements (shown as arbitrary units relative to PBGD) \pm SD. (C) Expression of Foxp3 (left panel) and TLR2 (right panel) protein determined by flow cytometry on resting PAM-expanded T cells (Tregs, gray lines; conventional $T$ helper cells, black lines) as well as freshly isolated $T$ cells (CD4+CD25+ Tregs, gray lines; CD4+CD25- Th cells, black lines). Corresponding isotype controls are indicated by the dotted lines. Representative results from 2 experiments are shown.

tional CD25- Th cells that were expanded using the same TLR2 ligand-based culture protocol did not exert any suppressive effects (Figure 6A). In addition, the supernatant of anti-CD3-activated Tregs did not transfer any suppressive effects, nor did we detect any cytokine production by these Tregs, using the mouse inflammation Cytometric Bead Array (not shown). Moreover, when placed behind a semipermeable membrane, the PAM-expanded Tregs failed to suppress Th proliferation, confirming that these Tregs mediate suppression via cell-cell contact (Supplemental Figure 3).

To address the functional quality of the PAM-expanded Tregs, we compared titrated amounts of freshly isolated WT and TLR2-/Tregs with in vitro-expanded PAM Tregs in an in vitro suppression assay. From the effective suppression of fresh Th cells by both WT and TLR2 $2^{--}$Tregs, we can conclude that there is no qualitative difference between WT and TLR2 $2^{--}$Tregs (Figure 6B). Moreover, the PAM-expanded WT Tregs were at least as efficient as the freshly isolated Tregs. Collectively, these data indicate that TLR2 triggering results in the expansion of Tregs that remain fully functional in the absence of TLR2 ligand.

TLR2 triggering on Tregs temporarily abrogates suppression in vitro. To address the functional consequences of TLR 2 triggering on Tregs, we performed suppression assays in the presence or absence of the TLR2 ligand PAM. To prove that any effects of TLR2 triggering on suppression are dependent on TLR2 signaling by Tregs and not on conventional T cells or APCs, we performed a suppression assay with both APCs and conventional CD4 ${ }^{+} \mathrm{T}$ cells isolated from TLR2 $2^{-/-}$mice but with freshly isolated WT (TLR2 $2^{++}$) Tregs. This setup ensured that TLR2 was solely expressed by the Treg subset and that all PAM-induced effects were caused via TLR2 signaling by Tregs. The results show that PAM induced some proliferation in the WT Tregs, although proliferation of Tregs remained approximately 15 -fold lower as compared with the TLR2-/- Th cells (Figure 7A). As expected, PAM had no effect on TLR2-/- Th cell proliferation. In Treg/Th cocultures, WT Tregs efficiently suppressed the proliferation of TLR2 ${ }^{-/-}$conventional Th cells. In contrast, addition of TLR2 ligand to the coculture completely abrogated suppression as observed by the restored TLR2 $2^{-/}$Th proliferation (Figure $7 \mathrm{~A}$ ). To exclusively monitor proliferation of the Th subset in Treg/Th coculture suppression assays, we used CFSE-labeled Th cells from TLR2-deficient mice and WT Tregs in a suppression assay similar to that described above. From the CFSE dilution profile (Figure 

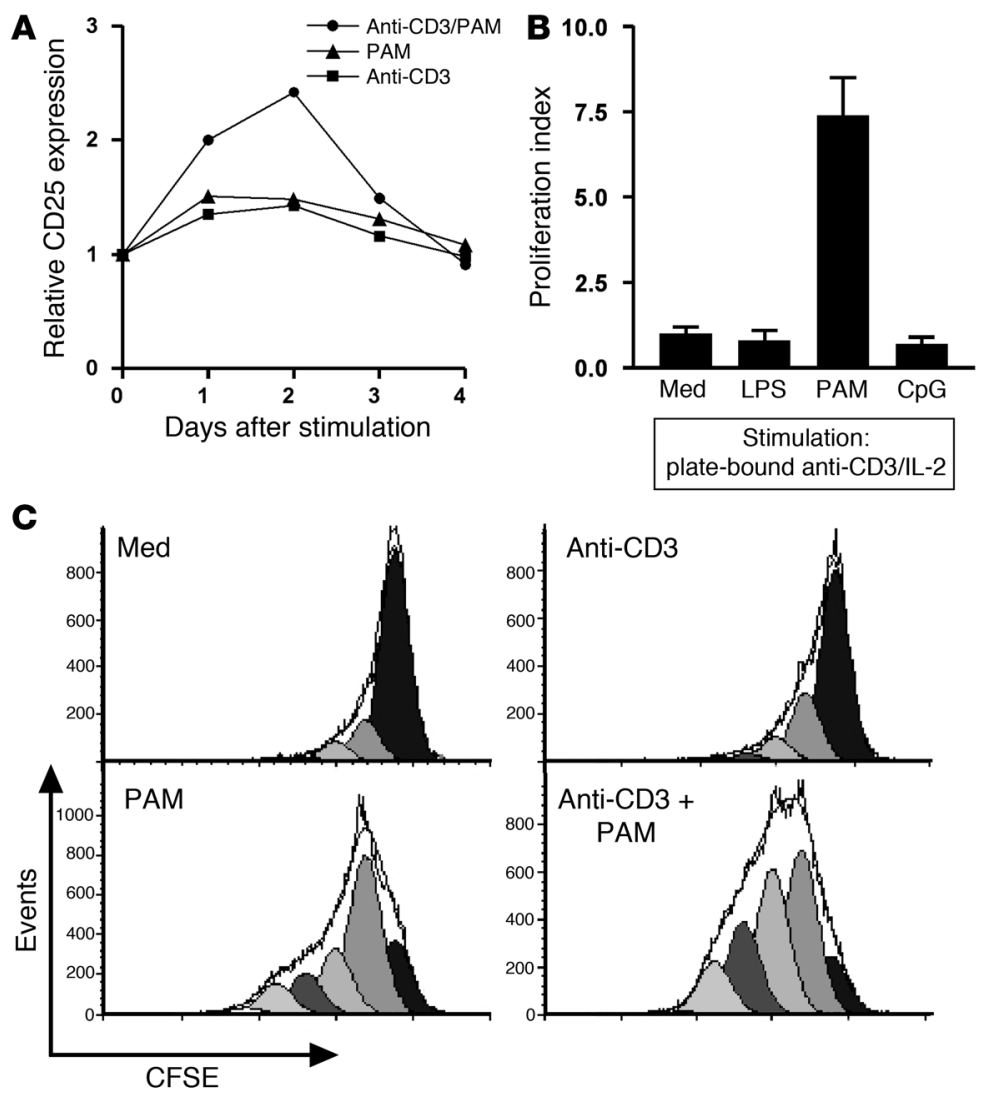

\section{Figure 5}

Proliferation of PAM-expanded Tregs. (A) TLR2 and TCR signals cooperate to increase CD25 expression on Tregs. Tregs were incubated with either PAM, anti-CD3, or a combination of both in IL-2-supplemented medium. CD25 expression was analyzed daily by flow cytometry and indicated as MFI relative to the medium control. (B) Proliferation of Tregs is induced by TLR2 signaling. PAM-cultured Tregs were stimulated on anti-CD3-coated plates with IL-2 (medium control) or with addition of the indicated TLR ligands. After 3 days, proliferation was measured by $\left[{ }^{3} \mathrm{H}\right]$ thymidine incorporation and shown as average $\mathrm{cpm}$ of triplicates relative to medium control \pm SD. (C) Proliferation of CFSE-labeled PAM-expanded Tregs. The labeled Tregs were cultured for 3 days in the presence of IL-2-supplemented medium or with the indicated stimulus (PAM and/or anti-CD3). Proliferation resulting in a decrease of fluorescent signal in the daughter cells was monitored by flow cytometry and (since in vitro-cultured T cell lines display a more broad signal after CFSE labeling compared with freshly isolated T cells) analyzed using ModFit software. Representative results from 3 experiments are shown.
$7 \mathrm{~B}$; the percentage of cells that proliferated $>3$ times is indicated), we can conclude that WT Tregs inhibit the Th proliferation in the coculture (to an extent similar to that reported before for this kind of analysis, ref. 17). The suppressive effect is, however, abrogated upon the addition of PAM. As expected, PAM had no effect on stimulated TLR2 $2^{-/}$Th cells. Moreover, when WT Tregs were pretreated overnight with anti-CD3 and PAM, extensively washed, and subsequently added to TLR2-/- Th cells in a coculture suppression assay, their suppressive ability was also abrogated (data not shown). Therefore, these results demonstrate that PAM-mediated TLR2 signaling on Tregs is responsible for the observed neutralization of their suppressive effect.

TLR2 induces Treg expansion in vivo. Interestingly, we observed that systemic PAM administration to WT mice resulted in an increase in $\mathrm{CD}^{+} \mathrm{Foxp}^{+} \mathrm{T}$ cell numbers (Supplemental Figure 1). This can be explained by a direct (see above) or indirect effect (for example, via APCs) of the TLR2 ligand.

To address the in vivo significance of the direct effects of TLR2 triggering on Treg expansion, TLR2-/- mice were reconstituted with freshly isolated and fluorescent-labeled (CFSE) OT-II transgenic Tregs. This setup ensured that the infused Tregs were the only TLR2 ligand responsive cells in these mice. The OT-II transgenic Tregs expressed a TCR $\left(V \alpha 2^{+}\right)$specific for a chicken OVA-derived peptide (chicken OVA-peptide) in the context of the murine MHC class II I-A ${ }^{\mathrm{b}}$. The OT-II Treg-reconstituted TLR2 $2^{-/-}$mice were subsequently challenged with either PAM or OVApeptide alone or with a combination of PAM and OVApeptide. Our results show that no significant proliferation of the infused Tregs was induced by either OVA-peptide or PAM alone (Figure 8A). In contrast, when PAM and OVA-peptide were combined, a significant part of the infused Tregs had proliferated
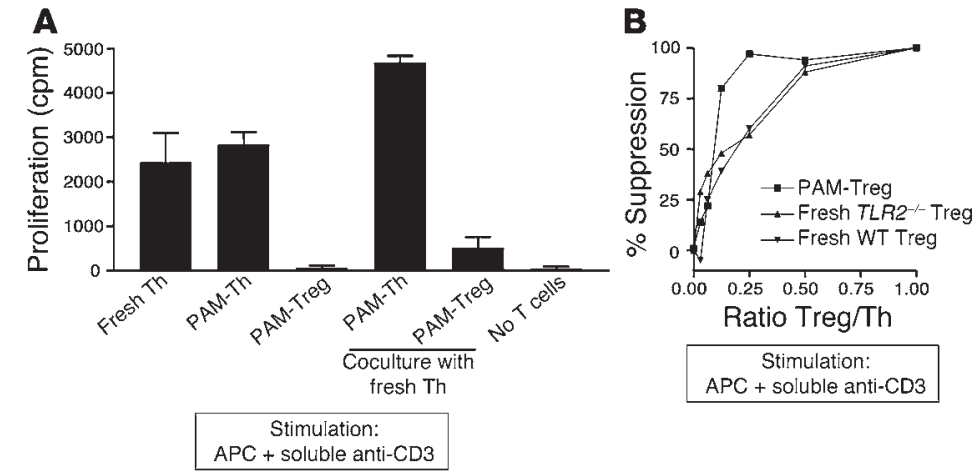

Figure 6

PAM-expanded Tregs remain suppressive. (A) In vitro suppression assay. PAM-expanded Tregs or control conventional Th cells $\left(0.5 \times 10^{4}\right)$ were rested for at least 5 days in the absence of TLR ligands and subsequently cocultured for 3 days with $10^{4}$ fresh naive CD4+ T cells, irradiated APCs, and anti-CD3. After 3 days, proliferation was measured and indicated as average cpm from triplicates \pm SD. Representative results from 3 experiments are shown. (B) Comparison of suppressive capacity of freshly isolated WT and TLR2-/- Tregs with PAM-expanded Tregs. Fresh naive CD4+ Th-cells $\left(2 \times 10^{4}\right)$ were cocultured with titrated numbers of Tregs. After 3 days, proliferation was measured by $\left[{ }^{3} \mathrm{H}\right]$ thymidine incorporation. Relative suppression was calculated with proliferation in the absence of Tregs (fresh CD4+ Th cells only) set at 0 and proliferation at the Treg/Th ratio of 1 at $100 \%$. Suppression/proliferation was measured from the average cpm in triplicate wells. 

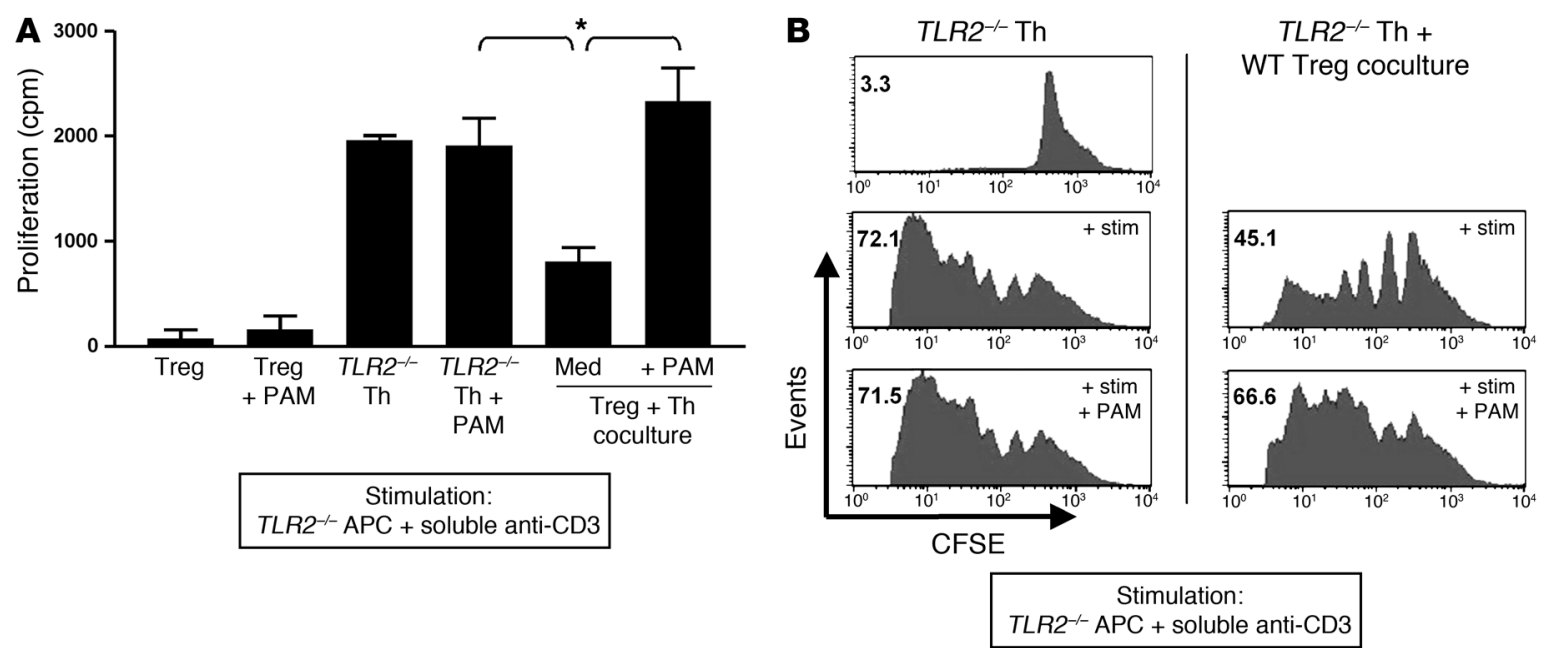

\section{Figure 7}

TLR2 controls Treg suppressor function in vitro. (A) To analyze the direct effects of TLR2 triggering on Treg suppressor function in vitro, $10^{4}$ $T L R 2^{--}$conventional T cells (Th) and $0.5 \times 10^{4}$ freshly isolated WT CD4 ${ }^{+}$CD25+ Tregs were (co)cultured for 3 days. Soluble anti-CD3 and irradiated $T L R 2^{-1-}$ APCs were used to stimulate the T cells, ensuring that TLR2 was solely expressed by Tregs. If indicated, PAM was added at the start of the coculture. Data indicate average proliferation from triplicates \pm SD. ${ }^{*} P<0.05$. (B) CFSE-labeled $T L R 2^{-/-}$Th $\left(10^{5}\right)$ were cocultured for 4 days with $0.5 \times 10^{5}$ WT Tregs as described in A. CFSE fluorescence intensity was measured by flow cytometry. Analysis was performed on all the CFSE ${ }^{+}$cells, using an exclusionary gate for the Treg subset (CFSE-negative CD25 high). The percentage of cells that divided more than 3 times is indicated. Representative results from 2 independent experiments are shown. Stim, stimulation.

( $75 \%$ versus $12 \%$ in the mice treated with PAM alone). We confirmed by flow cytometry that the proliferating $\mathrm{T}$ cells remained Foxp3 positive (data not shown). Thus, TCR and TLR2 signals cooperate to induce proliferation of Tregs in vivo by directly affecting Tregs themselves.

TLR2 modulates Treg function in vivo. To asses whether direct TLR2 signaling of Tregs in vivo can result in a modulation of Treg function, we used an acute fungal (C. albicans) infection model in which the kidney is the major fungal target organ (10). We have previously shown that Tregs inhibit the antifungal immune response as depletion of Tregs prior to a C. albicans challenge resulted in decreased C. albicans outgrowth from the kidney (10) and increased IFN- $\gamma$ production by splenocytes (10). Applying this infection model in TLR2 $2^{-1-}$ mice reconstituted with $4 \times 10^{6}$ syngeneic WT Tregs, we ensured that all effects of PAM administration must be caused by TLR 2 triggering of the infused Tregs since these are the only TLR2-expressing cells in this system. The WT Treg reconstituted mice were challenged with an i.v. injection of live C. albicans, and we monitored $C$. albicans outgrowth in the presence or absence of TLR2 ligand administration. The results showed that the WT Tregreconstituted $T L R 2^{-/-}$mice exhibited a 2 log increase in C. albicans outgrowth compared with the nonreconstituted TLR2-/- controls (Figure $8 \mathrm{~B}$ ), indicating that the infused Tregs are potent inhibitors of the anti-C. albicans immune response. Strikingly, administration of TLR2 ligand to the WT Treg-reconstituted mice restored the level of $C$. albicans outgrowth to the level of the nonreconstituted TLR2 ${ }^{-/-}$mice (Figure 8B), indicating that the TLR2 trigger abrogated the suppressive effects of the infused Tregs in vivo. The administration of TLR 2 ligand alone to the TLR2-/- mice did not affect the C. albicans outgrowth (not shown). In addition, the administration of PAM-expanded conventional Th cells $\left(4 \times 10^{6}\right.$ with or without PAM) did not affect the C. albicans outgrowth in the TLR2 $2^{--}$mice (Figure 8C). This shows that Tregs, but not conventional Th cells, are able to inhibit the immune response against C. albicans. More- over, IFN- $\gamma$ production by ex vivo C. albicans-stimulated splenocytes was analyzed. C. albicans-stimulated splenocytes of the Treg-infused $T L R 2^{-1-}$ mice produced significantly less IFN- $\gamma$ compared with TLR2 ${ }^{-/-}$controls (Figure 8D). Moreover, this suppression of IFN- $\gamma$ production was absent in splenocytes from mice that received both Tregs and TLR2 ligands. Of note, TLR2 ligand alone had no effect on cytokine production by TLR2 $2^{--}$splenocytes ex vivo (not shown). The above-described results demonstrate that TLR2 triggering abrogated the suppressive capacity of the infused Tregs in vivo. Since the infused Tregs were the only TLR2-expressing cells in this in vivo system, the abrogation of suppression can only be explained by a direct effect of the TLR 2 ligand on the infused TLR2 ${ }^{+/+}$Tregs in the TLR2 $2^{-1-}$ mice.

Our combined results show that the suppressive function of Tregs is directly controlled by the pathogen-associated molecular pattern receptor TLR2. In the presence of TLR2 ligand, Treg expansion and a temporal loss of suppression is observed. After removal of TLR2 ligand, the expanded Tregs regain their suppressive capabilities.

\section{Discussion}

The identification of $\mathrm{CD} 25^{\text {high }} \mathrm{CD} 4^{+} \mathrm{T}$ cells as $\mathrm{T}$ cells bearing a suppressive phenotype has renewed interest in Tregs (5). These Tregs are now emerging as major regulators of our immune systems. Lack of Tregs results in various autoimmune syndromes. On the other hand, Treg-mediated suppression might hinder the development of effective immune responses, which are crucial for the elimination of tumors and infections. Therefore, the regulators need to be well controlled. We now demonstrate for what we believe is the first time that TLR2 triggering by PAMPs present on or secreted by infectious organisms can directly modulate Treg function.

Our initial observation that $\mathrm{CD} 4^{+} \mathrm{CD} 25^{+} \mathrm{T}$ cell numbers were decreased in TLR2-deficient but not in TLR4-deficient mice led us to investigate the role of TLR2 signaling on $\mathrm{CD} 4{ }^{+} \mathrm{CD} 25^{+} \mathrm{T}$ cell functionality in more detail. Analysis of blood and spleen of 
A
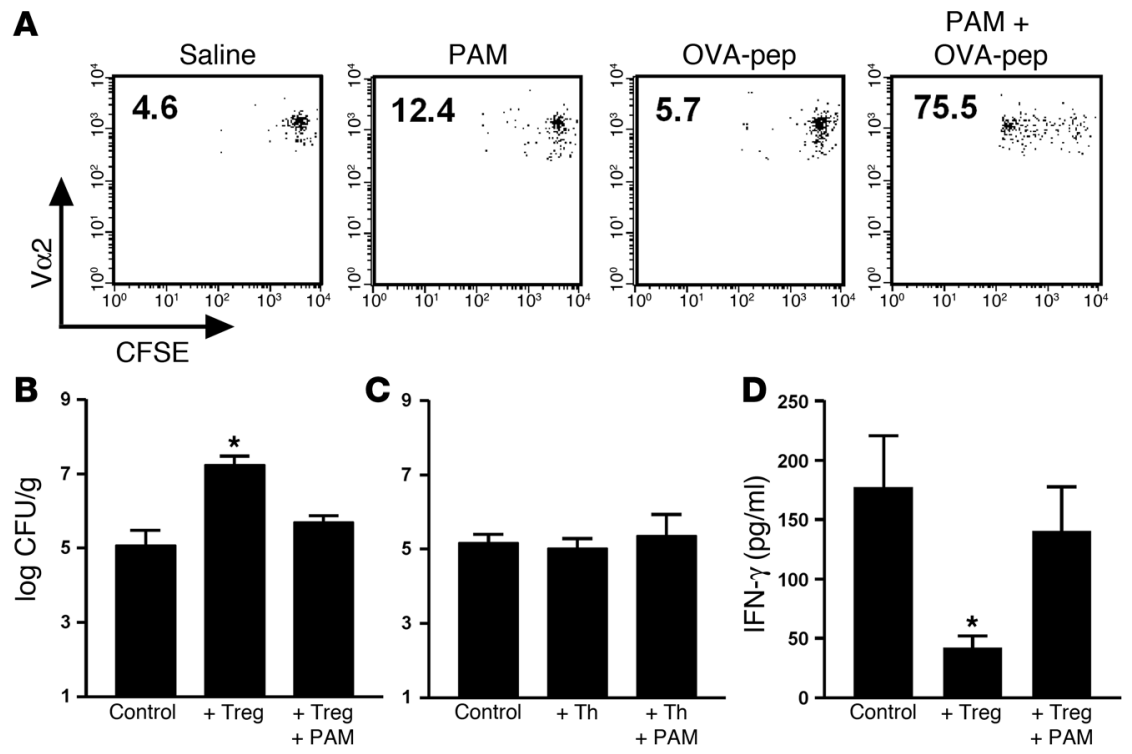

Figure 8

TLR2 controls Treg suppressor function in vivo. (A) TLR2 and TCR triggering cooperate to induce Treg expansion in vivo. TLR2 $2^{-/-}$mice were reconstituted with $2 \times 10^{6}$ freshly isolated and CFSE-labeled OT-II-transgenic Tregs (TCR of OT-II transgenic T cells is $\mathrm{V} \alpha 2$ and specific for the OVA-peptide presented in $\left.I-A^{b}\right)$. The reconstituted mice were subsequently challenged i.p. with either PAM (20 $\mu \mathrm{g} /$ mouse) or OVA-peptide [OVA-pep] (10 $\mu \mathrm{g} / \mathrm{mouse})$ alone or with the combination of PAM and OVA-peptide. After 4 days, splenocytes were isolated and analyzed by flow cytometry for CFSE-fluorescent signal of the infused cells. The cells shown are gated for the $\mathrm{CD}^{+}, \mathrm{V}_{2} 2^{+}, \mathrm{CFSE}^{+}$cells, and propidium iodide-positive (death) cells were excluded from the analysis. The value indicates the percentage of cells within the proliferative fraction (>1 division). (B and C) TLR2 triggering abrogates Treg-mediated suppression of anti-C. albicans immunity in vivo. $T L R 2^{-/-}$mice (5 per group) were reconstituted with $4 \times 10^{6}$ WT PAM-expanded Tregs (B) or conventional Th cells (C) and challenged i.v. with $10^{5}$ live $C$. albicans cells 1 day later (day 0). If indicated, mice received an i.p. injection of 100 $\mu \mathrm{l}$ saline (controls) or $20 \mu \mathrm{g}$ PAM/100 $\mu$ l saline on days $-1,1,3$, and 5 . SEVEN days after the challenge, $C$. albicans outgrowth (CFU/g tissue \pm SEM) from kidneys was monitored. (D) Ex vivo IFN- $\gamma$ production ( \pm SEM) by $C$. albicans-stimulated splenocytes was measured as described in Methods. Representative results of 2 independent experiments are shown. ${ }^{*} P<0.05$ with $T L R 2^{-/}$control.

MyD88-deficient mice revealed that, in addition to TLR2, MyD88 is also required for normal $\mathrm{CD} 4{ }^{+} \mathrm{CD} 25^{+} \mathrm{T}$ cell levels in vivo (Figure 1). Moreover, massive TLR2 triggering by i.v. injection of the TLR2 ligand PAM had the opposite effect and resulted in a significant proliferation and increase of $\mathrm{CD} 4{ }^{+} \mathrm{CD} 25^{+} \mathrm{T}$ cell numbers (Supplemental Figure 1).

The in vivo increase of $\mathrm{CD} 4^{+} \mathrm{CD} 25^{+} \mathrm{T}$ cells by the TLR2 ligand PAM might be caused by an indirect effect via APCs or by direct TLR2 triggering of Tregs. In vitro, we found that all TLR ligands tested increased Treg proliferation in the presence of irradiated APCs and anti-CD3 (Figure 2). However, our results also demonstrate that the TLR2 ligand PAM, but not purified LPS or CPG, is able to induce proliferation of Tregs in the absence of APCs by directly affecting Tregs themselves. Next to PAM, MALP-2, peptidoglycan, and heat-killed C. albicans also resulted in Treg activation, emphasizing that besides synthetic ligands, natural TLR2 ligands can also modulate Tregs. Using the TLR2 ligand PAM, we were able to establish long-lived (up to 5 months), proliferating Treg lines. Although the exact mechanism remains to be elucidated, the finding that TLR 2 triggering of Tregs strongly enhances CD25 expression implies that increased sensitivity to IL-2 might be involved.
The in vitro-expanded Tregs expressed CD4, CD25, GITR, CTLA-4, CD103, and Foxp3 (Figure 4), all typical Treg markers $(1,21)$. These suppressor $\mathrm{T}$ cell lines further expressed significant amounts of the PAM receptor TLR2 (Figure 4) but also remained sensitive to TLR2 stimulation, further emphasizing the direct interaction between TLR2 ligands and Tregs (Figure 5). Furthermore, they fully maintained their suppressive capacities following withdrawal of TLR2 ligands (Figure 6). Interestingly, TLR2 $2^{--}$Tregs expressed the same surface markers as WT Tregs and were equally efficient in the suppression of naive $T$ cell responses (Figure 6 and data not shown). This indicates that TLR2 is not involved in the suppression process itself. We additionally showed that the effects of TLR2 triggering on Tregs temporarily abrogated their suppressive capabilities as well (Figure 7). We hypothesize that TLR2 induces proliferation of Tregs, during which there is a temporal loss of the suppressive phenotype.

The physiological relevance of our findings was assessed by infusion of TLR2 $2^{+/}$Tregs into $T L R 2^{-/-}$mice, allowing for the analysis of direct TLR2 effects on Treg function in vivo. Importantly, the combination of TCR and TLR2 signals induced strong proliferation of Tregs in vivo (Figure 8A), confirming that TLR2 and TCR signals cooperate in the proliferation of Tregs. Furthermore, in line with our in vitro observations, infused WT Tregs inhibited antifungal immune responses in TLR2 $2^{-/-}$mice, while coadministration of TLR2 ligands resulted in a loss of the suppressive phenotype of the infused Tregs as observed by the restored antifungal immunity (Figure 8, B-D).

Recently, it was found that IL-2 (3) and strong TCR signals (13) release effector $\mathrm{T}$ cells from Treg-mediated suppression. Furthermore, the presence of the TLR9 ligand CPG in a suppression assay containing APCs resulted in a reduced suppression by Tregs. This is due to TLR9-triggered production of IL- 6 by the APCs rendering conventional $\mathrm{T}$ cells insensitive to Treg-mediated suppression (12). Another report nicely links TLR4 and TLR9 signaling with Treg-mediated CD8 tolerance (25). The authors demonstrate that ligation of TLR4 or TLR9 on DCs is required for breaking Treg-mediated CD8 tolerance in vivo. However, in addition to the effects of TLR ligands on DCs, the results described here show that it is also possible that a TLR ligand directly affects Tregs, resulting in abrogation of suppression and expansion of the Treg subset. A study by Komai-Koma et al. (26) showed that TLR2 triggering also stimulates activated, conventional $\mathrm{T}$ cells. We were able to confirm their observations on activated conventional CD4 ${ }^{+} \mathrm{T}$ cells (data not shown). However, using TLR2-deficient conventional T cells, we demonstrate that it is the Treg subset (freshly isolated or in vitro expanded) that is highly sensitive for TLR2 stimulation, resulting in profound proliferation and reduced suppression by Tregs. The effects of TLR ligands in vivo might thus be more 


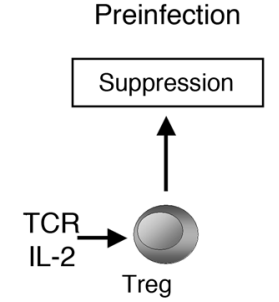

No/low exogenous TLR2 ligand

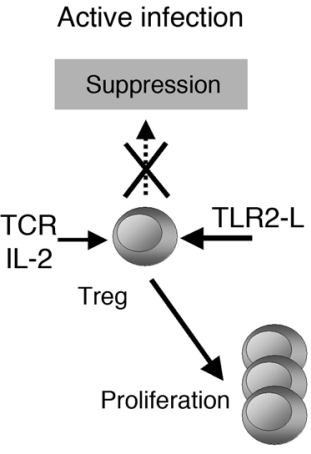

High exogenous TLR2 ligand

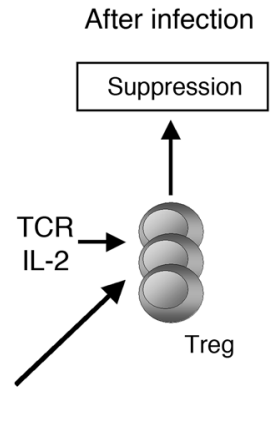

No/low exogenous TLR2 ligand

\begin{abstract}
Figure 9
Model of TLR2-mediated control of Treg function. In a preinfection setting, Treg function is mainly regulated by TCR stimulation and IL-2. During an acute infection, pathogen-derived TLR2 ligands promote proliferation of Tregs paralleled by temporarily abrogated suppression. As a result, Tregs do not suppress the ongoing immune response. Once the pathogen is cleared by the immune system, the source of TLR2 ligands is no longer present, and Tregs will regain their suppressive capabilities, thus contributing to the balance between tolerance and immunity.
\end{abstract}

complicated than initially thought, affecting multiple cell types of both the innate and the acquired immune system.

Recently, a role for TLR4 on Treg homeostasis was reported (19). We and others $(17,26)$, however, did not find any effects of purified LPS on T cells (conventional or regulatory). We note that in contrast to purified LPS, commercially obtained LPS (containing contaminating TLR2 ligands; ref. 27) induced a significant CD25 upregulation on Tregs (Supplemental Figure 2B). Thus, to exclude bacterial contamination in all our experiments, we only used purified LPS and synthetic TLR2 and TLR9 ligands. Moreover, we confirmed the purity of our TLR2 and TLR4 ligands by measuring their activation capacity on TLR2- and TLR4-deficient DCs (Supplemental Figure 4).

An intriguing question that remains to be answered is why TLR2 but not TLR4 is able to directly affect Tregs. TLR2 but not TLR4 is found to promote IL-10 production by innate immune cells in vitro (28). The accumulating evidence that TLR2 is also involved in immunosuppression in vivo (reviewed in ref. 29) renders TLR2 unique compared with other TLRs. In this context, it is interesting to note a report linking the antiinflammatory glucocorticoids with TLR2 expression (30). These observations strengthen the idea that TLR2, besides having an activating function, could be involved in tolerance as well by acting directly on APCs and Tregs. In addition, other TLRs not tested here could act on Tregs. It was recently shown that TLR8 can abrogate the suppressor function of human Tregs (31). Although TLR8 did not induce proliferation of human Tregs, this report strengthens our findings of TLR2-mediated control of murine Tregs. We are now investigating the possibility that different TLR signals can differentially modulate Treg function.

Our finding of the abrogation of suppression after TLR2 stimulation is in line with reports indicating that Tregs rapidly lose their ability to inhibit proliferation after receiving strong activation signals (13). In addition, Komai-Koma reported that TLR2 functions as a costimulatory molecule for conventional $\mathrm{T}$ cells, resulting in increased T cell expansion after TLR2 triggering on the conventional T cells (26). Furthermore, the finding that TLR8 triggering combined with TCR stimulation on human Tregs resulted in reversal of their suppressive capabilities emphasizes the effect of TLR signaling on Treg-mediated suppression (31). In line with these reports and our own observations, we envisage that TLR2 signaling on murine Tregs contributes to the strength of signals received by Tregs. This forces Tregs into the proliferative pathway, which is paralleled by a reversal of suppressive capabilities. Once in a resting state again, Tregs regain their suppressive capacities (see also Figure 9).
Our observation that PAMPs can directly act on Tregs provides new insights regarding the role of Tregs in the induction of immune responses. The results presented here provide evidence for a strict control of Tregs by TLR2 ligands. The current opinion holds that TLR-mediated recognition of pathogens results in DC activation and subsequent initiation of $\mathrm{T}$ cell responses. We can now add a direct Treg modulating capacity to TLR ligands. Applying an acute fungal infection model, we unequivocally demonstrate that TLR2 triggering on Tregs abrogates their suppressive activity in vivo, resulting in increased IFN- $\gamma$ production and decreased fungal outgrowth. These data imply that TLR2 ligands provided by a microbial invasion during acute infection mediate Treg expansion and abrogation of Treg-mediated suppression, thus allowing a potent immune response to occur. However, after infection, when the immune system has cleared the pathogen and hence TLR2 ligands are declining, the expanded Tregs regain their suppressive activity and could help to restore the immune balance (see also schematic model in Figure 9). Interestingly, 2 recent reports suggested that $\mathrm{CD} 4{ }^{+} \mathrm{CD} 25^{+}$Tregs control the persistence and memory protective immunity against Leishmania major and C. albicans (9, 32). Based on the novel findings described herein, one could envisage that TLR2 ligands provided by the pathogens (33) could first expand Tregs in vivo and abrogate their suppressive phenotype. When only low numbers of the pathogen are present, as in the persistent phase of $L$. major infection, Tregs regain their suppressive phenotype and could be responsible for pathogen persistence.

Finally, we note that the presence of microorganisms in the gut might contribute to a constant influx of TLR ligands that can modulate Treg numbers. In support of this hypothesis is our unpublished observation that germ-free mice have altered Treg numbers as compared with control mice. In addition to microbial-derived PAMPs, TLR2 signaling can possibly be induced by endogenous proteins (e.g., heat shock proteins; reviewed in ref. 34). This would allow the modulation of the Treg compartment in a nonpathogenic, stress-induced (anti)inflammatory environment.

Collectively, our findings have major implications for our understanding of immune regulation by both Tregs and TLRs. Ultimately, the knowledge that TLRs can be used to expand and modulate Tregs will lead to new methods for Treg-based immunotherapy of diseases.

\section{Methods}

Mice. C57BL/6 mice were obtained from Charles River WIGA Gmbh. TLR2-(35), $T L R 4^{-/-}$, and $M y D 88^{-/-}$(36) mice were obtained from S. Akira (Osaka University, Osaka, Japan). The MyD88-/- mice were backcrossed more than 
8 times on the C57BL/ 6 background. The OVA-specific TCR transgenic OT-II mice (on a C57BL/6 background) were obtained from Jackson Laboratory. The MyD88-KO mice were bred in our specified pathogen-free animal facility using heterozygous breeding pairs. The WT littermates were used as controls for the comparison of Treg numbers. All animal experiments were approved by the Animal Experimental Committee of Radboud University Nijmegen Medical Centre and were performed in accordance with institutional and national guidelines.

Antibodies and flow cytometry. Directly labeled monoclonal antibodies used for staining by anti-CD3-PE, anti-CD4-APC, anti-Va2-PE, anti-CD25FITC (clone 7D4), anti-rat-PE and all isotype controls were obtained from BD Biosciences - Pharmingen. Anti-mTLR2-PE (clone T2.5) and antimFoxp3-PE (clone FJK; staining according to instructions by manufacturer) were obtained from eBioscience. Anti-hamster-APCs were obtained from CALTAG Laboratories. In addition, anti-GITR (DTA-1, a kind gift from S. Sakaguchi, Kyoto University, Kyoto, Japan, anti-CTLA-4 (clone 9H10), anti-CD86, and anti-CD103 (BD Biosciences - Pharmingen) were used. Analysis of cell surface markers on lymphocytes was performed using a FACScalibur (BD) and CELLQuest software (version 3.3; BD Biosciences - Pharmingen). For analysis of relative $\mathrm{CD} 4{ }^{+} \mathrm{CD} 25^{+} \mathrm{T}$ cell numbers in vivo, 50-100 $\mu \mathrm{l}$ blood was collected in heparin-coated tubes, and erythrocytes were lysed using standard protocols. The remaining lymphocytes were washed and incubated with anti-CD25-FITC and anti-CD4-APCs and subsequently analyzed on a flow cytometer

$T$ cell purification and analysis. Spleens from WT or KO mice were mashed and filtered, and $\mathrm{CD} 4{ }^{+} \mathrm{T}$ cells were purified using anti-mouse-CD4 Microbeads (MACS, Miltenyi Biotec), resulting in a $95 \%$ pure $\mathrm{CD}^{+} \mathrm{CD}^{+} \mathrm{T}$ cell population, as measured by flow cytometry. $\mathrm{CD} 4^{+} \mathrm{CD} 25^{-}$and $\mathrm{CD} 4^{+} \mathrm{CD} 25^{+}$ $\mathrm{T}$ cell subsets were obtained by flow cytometry purification of the presorted $\mathrm{CD} 4{ }^{+} \mathrm{T}$ cells; $\mathrm{CD} 4$ cells were stained with APC-conjugated CD4 mAb and FITC-conjugated CD25. For exclusion of MHC class $\mathrm{II}^{+}$cells and $\mathrm{B}$ cells, anti-I-A $\mathrm{A}^{\mathrm{b}}(17 / 227$, mouse IgG2a) and PE-conjugated goat anti-mouse IgG were used. Cell sorting was performed on a Coulter Altra HyPerSort cell sorter. The purity of each cell preparation was greater than $98 \%$. Purified $\mathrm{CD}^{+} \mathrm{T}$ cell subsets were subsequently cultured for 3 days in complete medium (Iscoves IMDM [Invitrogen Corp.], 9\% FCS, $2 \mu \mathrm{M}$ L-glutamine, $50 \mu \mathrm{M} \beta$-mercapthoethanol, and 1\% antibiotic-antimycotic [Invitrogen Corp.]) with $1 \mu \mathrm{g} / \mathrm{ml}$ anti-CD3 (BD Biosciences - Pharmingen) and 5 Cetus units (cU; $1 \mathrm{cU}=5 \mathrm{IU}) \mathrm{IL}-2 / \mathrm{ml}$ with or without TLR ligands. Unless indicated otherwise, TLR ligands were used at the following concentrations: PAM and MALP-2, both $2 \mu \mathrm{g} / \mathrm{ml}$ (EMC Microcollections); nonpurified and purified E. coli LPS, $10 \mu \mathrm{g} / \mathrm{ml}$ (obtained from Sigma-Aldrich and subsequently purified as described in ref. 27); peptidoglycan, $10 \mu \mathrm{g} / \mathrm{ml}$ (Sigma-Aldrich); purified LTA, $10 \mu \mathrm{g} / \mathrm{ml}$ (obtained from T. Hartung, University of Konstanz, Konstanz, Germany); heat-killed C. albicans hyphe and conidia, $2 \times 10^{4} \mathrm{CFU}$ per well of 96-well plate (strain UC 820); and

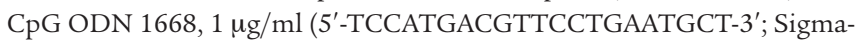
Aldrich). After 3 days of culture, the cells were harvested, stained with APCconjugated anti-CD4 and FITC-conjugated anti-CD25, and analyzed. Data indicate mean fluorescence intensity (MFI) presented as the average MFI of 3 measurements with SD indicated.

Treg culture and suppression assay. Purified $\mathrm{CD} 4^{+} \mathrm{CD} 25^{+} \mathrm{T}$ cells, $10^{4}$ per well of a 96-well plate, were stimulated weekly with $10^{5}$ irradiated APCs (CD4-MACS bead depleted splenocytes), $2 \mu \mathrm{g} / \mathrm{ml}$ PAM, $1 \mu \mathrm{g} / \mathrm{ml}$ anti-CD3 (145-2C11; BD Biosciences - Pharmingen), and $20 \mathrm{cU}$ IL-2/ml complete medium. The cells were washed 3 days after stimulation and maintained in culture medium supplemented with $20 \mathrm{cU}$ IL-2/ml. When necessary, dead cells were removed by ficol density gradient. Cultured Tregs were used in assays at least 6 days after stimulation. Bone marrow cells were cultured for 7 days in complete medium in the presence of $20 \mathrm{ng} / \mathrm{ml} \mathrm{mIL-4}$ and
mGM-CSF (PrepoTech) to obtain bone marrow-derived DCs. Suppression assays were performed as follows: freshly sorted $\mathrm{CD} 4^{+} \mathrm{CD} 25^{-}$naive $\mathrm{T}$ cells $\left(20 \times 10^{3}\right)$ and either cultured or freshly isolated Tregs $\left(5 \times 10^{3}\right)$ were mixed and cocultured for 3 days. If indicated, the T cells were stimulated with TLR ligands and with either plate-bound anti-CD3 $(2.5 \mu \mathrm{g} / \mathrm{ml})$ or soluble anti-CD3 $(1 \mu \mathrm{g} / \mathrm{ml})$ plus $10^{5}$ irradiated APCs in complete medium. In a number of coculture experiments, resting TLR2 $2^{-/} \mathrm{CD} 25^{-}$conventional $\mathrm{T}$ cells were used as responders. These cells were maintained in culture for a maximum of 6 weeks, using weekly restimulation assays with irradiated APCs, anti-CD3 $(1 \mu \mathrm{g} / \mathrm{ml})$, and $20 \mathrm{cU}$ IL-2/ml. After 3 days of coculture, supernatant was collected for cytokine analysis using a mouse inflammation CBA kit (BD Biosciences). Proliferation was measured by overnight (20 hours) $\left[{ }^{3} \mathrm{H}\right]$ thymidine incorporation. In addition, suppression/proliferation was monitored by analysis of CFSE-labeled $(1 \mu \mathrm{M}) \mathrm{T}$ cells. CFSE-labeled cells $\left(10^{5}\right)$ were cultured with the indicated stimulus for 4 days, and subsequently, CFSE fluorescence intensity was measured by flow cytometry. The transwell-suppression assay was performed in a 24-well culture plate (Corning), using $10^{6}$ freshly isolated CFSE-labeled $\mathrm{CD}^{+} \mathrm{T}$ cells cocultured with an equal number of Tregs or with Tregs behind a semipermeable membrane $(0.4 \mu \mathrm{m}$; Millipore). The $\mathrm{T}$ cells were stimulated with $10^{6}$ irradiated APCs (also in the upper chamber of the transwell) and $1 \mu \mathrm{g} / \mathrm{ml}$ anti-CD3 in a total volume of $1 \mathrm{ml}$ culture medium. For monitoring proliferation of in vitro-expanded Tregs by CFSE-fluorescence (in vitro-expanded T cells displayed a broad CFSE signal as compared with freshly isolated T cells), we made use of ModFit LT software (version 3.0; Verity Software House) to illustrate the number of daughter generations after 4 days of proliferation.

Quantitative PCR. Relative mRNA levels were determined using the ABI PRISM 7000 Sequence Detection System and SYBR Green Reagent (Applied Biosystems; 4309155). cDNAs were synthesized from $2.0 \mu \mathrm{g}$ of total RNA using Moloney murine leukemia virus reverse transcriptase (Invitrogen Corp.; 28025-013). PRISM samples contained $1 \times$ SYBR Green Master Mix, $1.5 \mu \mathrm{l} 5 \mu \mathrm{M}$ primers, and $25 \mathrm{ng}$ synthesized cDNA in a $25-\mu \mathrm{l}$ volume. The following primers were used: mTLR2 forward (5'-AACCTCAGACAAAGCGTCAAATC-3'), mTLR2 reverse (5'-ACCAAGATCCAGAAGAGCCAAA-3') (both from Biolegio bv); mFOXP3 forward (5'-AGGAGAAGCTGGGAGCTATGC-3'), mFOXP3 reverse (5'-GGTGGCTACGATTGCAGCAA- $3^{\prime}$ ); porphobilinogen deaminase (PBGD) forward (5'-CCTACCATACTACCTCCTGGCTTTAC-3'), PBGD reverse (5'-TTTGGGTGAAAGACAACAGCAT-3') (all from Sigma-Aldrich). Mean relative mRNA expression from 3 replicate measurements was calculated using ABI PRISM 7000 SDS software (version 1.0; Applied Biosystems). Values are expressed as arbitrary units relative to PBGD.

In vivo Treg proliferation. Spleen and inguinal lymph nodes from OT-II transgenic mice were isolated and mashed into single-cell suspension. Subsequently, the cells were stained with anti-CD25-FITC and isolated using anti-FITC MACS beads (Miltenyi Biotec). Contaminating CD8 ${ }^{+}$ $\mathrm{T}$ cells and B cells were removed using negative depletion with Dynal beads. In this way, up to $95 \%$ pure $\mathrm{CD} 4{ }^{+} \mathrm{CD} 25^{+} \mathrm{T}$ cells were obtained. The OT-II transgenic Tregs were CFSE labeled $(5 \mu \mathrm{M})$ and injected i.p. into TLR2 ${ }^{-/-}$mice $\left(2 \times 10^{6}\right.$ per mouse). Four hours later, mice were challenged i.p. with $20 \mu \mathrm{g} /$ mouse PAM and/or $10 \mu \mathrm{g} /$ mouse OVA-peptide (ISQAVHAAHAEINEAGR, obtained from J.W. Drijfhout, Leiden University Medical Centre, Leiden, The Netherlands; peptide was synthesized by standard Fmoc chemistry, and purity [95\%] was checked by HPLC). Four days later, the mice were sacrificed, spleens were isolated, and the splenocytes were stained with anti-CD4-APCs, anti-V 2 2-PE, and propidium iodide and analyzed by flow cytometry. In addition, the cells were stained with anti-Foxp3-PE and CD4-APCs to confirm that the CFSE ${ }^{+}$ cells were still Foxp $3^{+}$. 
C. albicans infection model. We used a nonlethal C. albicans infection model as described (10). Briefly, $10^{5}$ CFUs of C. albicans strain UC 820 were injected i.v. in mice on day 0 . The mice, 5 per group, received, if indicated, $2 \times 10^{6}$ WT in vitro-expanded Tregs or Th cells i.v. in $100 \mu \mathrm{l}$ saline on days -2 and -1 ( $4 \times 10^{6}$ in total per mouse). On days $-1,1,3$, and 5 , all mice received 100 $\mu \mathrm{l}$ saline or $20 \mu \mathrm{g} \mathrm{Pam}{ }_{3} \mathrm{Cys} / 100 \mu \mathrm{l}$ saline. At day 7 , kidneys and livers were aseptically removed, weighed, and homogenized in sterile saline in a tissue grinder. The number of viable C. albicans cells was determined by plating serial dilutions on Sabouraud dextrose agar plates. The colonies were counted after 24 hours at $37^{\circ} \mathrm{C}$ and indicated as CFU/g tissue. The spleens were also isolated from the mice and were used for the analysis of cytokine production in response to heat-killed C. albicans. Splenocytes $\left(5 \times 10^{6}\right.$ in $1 \mathrm{ml}$ complete medium) were stimulated with $10^{7}$ heat-killed C. albicans cells. The supernatants were collected after 48 hours of incubation at $37^{\circ} \mathrm{C}$ in $5 \% \mathrm{CO}_{2}$, and measurement of IFN- $\gamma$ in the supernatant was performed by a commercial ELISA (BioSource International; detection limit $16 \mathrm{pg} / \mathrm{ml}$ ), according to the instructions of the manufacturer.

Statistics. Statistical analysis was performed with Student's $t$ test using 2-tailed distribution.

\section{Acknowledgments}

We thank A. Pennings (deceased; Centraal Hematologisch Laboratorium, Radboud University Nijmegen Medical Centre) for the FACSsort purification of T cell subsets. We thank Shimon Sakaguchi for the DTA-1 hybridoma. We thank M.E. Morgan, C.G. Figdor, and C. Punt (Radboud University Nijmegen Medical Centre) for critically reading the manuscript. This research is supported in part by grants from the Dutch Cancer Society (KWF 2003-2893 to R.P.M. Sutmuller and 2005-3325 to M.H.M.G.M. den Brok) and from The Netherlands Organization for Scientific Research (VENI grant to R.P.M. Sutmuller and VIDI grant to M.G. Netea).

Received for publication April 22, 2005, and accepted in revised form November 15, 2005.

Address correspondence to: G.J. Adema, Tumor Immunology Laboratory, Radboud University Nijmegen Medical Centre, P.O. Box 9101, 6500 HB Nijmegen, The Netherlands. Phone: 31-243617600; Fax: 31-24-3540339; E-mail: G.Adema@ncmls.ru.nl.
1. Sakaguchi, S. 2004. Naturally arising CD4+ regulatory $\mathrm{T}$ cells for immunologic self-tolerance and negative control of immune responses. Annu. Rev. Immunol. 22:531-562.

2. Shevach, E.M. 2002. CD4+ CD25+ suppressor T cells: more questions than answers. Nat. Rev. Immu nol. 2:389-400.

3. Thornton, A.M., and Shevach, E.M. 2000. Suppressor effector function of CD4+CD25+ immunoregulatory $\mathrm{T}$ cells is antigen nonspecific. J. Immunol. 164:183-190.

4. Piccirillo, C.A., and Shevach, E.M. 2001. Cutting edge: control of CD8+ $\mathrm{T}$ cell activation by CD4+CD25+ immunoregulatory cells. J. Immunol. 167:1137-1140.

5. Sakaguchi, S., Sakaguchi, N., Asano, M., Itoh, M., and Toda, M. 1995. Immunologic self-tolerance maintained by activated T cells expressing IL-2 receptor alpha-chains (CD25). Breakdown of a single mechanism of self-tolerance causes various autoimmune diseases. J. Immunol. 155:1151-1164.

6. Itoh, M., et al. 1999. Thymus and autoimmunity: production of CD25+CD4+ naturally anergic and suppressive $\mathrm{T}$ cells as a key function of the thymus in maintaining immunologic self-tolerance. J. Immunol. 162:5317-5326.

7. Shevach, E.M. 2000. Regulatory T cells in autoimmmunity*. Annu. Rev. Immunol. 18:423-449.

8. Morgan, M.E., et al. 2003. CD25+ cell depletion hastens the onset of severe disease in collageninduced arthritis. Arthritis Rheum. 48:1452-1460.

9. Montagnoli, C., et al. 2002. B7/CD28-dependent $\mathrm{CD} 4+\mathrm{CD} 25+$ regulatory $\mathrm{T}$ cells are essential components of the memory-protective immunity to Candida albicans. J. Immunol. 169:6298-6308.

10. Netea, M.G., et al. 2004. Toll-like receptor 2 suppresses immunity against Candida albicans through induction of IL-10 and regulatory T cells. J. Immunol. 172:3712-3718.

11. Sutmuller, R.P., et al. 2001. Synergism of cytotoxic $\mathrm{T}$ lymphocyte-associated antigen 4 blockade and depletion of $\mathrm{CD} 25(+)$ regulatory $\mathrm{T}$ cells in antitumor therapy reveals alternative pathways for suppression of autoreactive cytotoxic T lymphocyte responses. J. Exp. Med. 194:823-832.

12. Pasare, C., and Medzhitov, R. 2003. Toll path- way-dependent blockade of CD4+CD25+ T cellmediated suppression by dendritic cells. Science. 299:1033-1036.

13. Baecher-Allan, C., Viglietta, V., and Hafler, D.A. 2002. Inhibition of human CD4(+)CD25(+high) regulatory $\mathrm{T}$ cell function. J. Immunol. 169:6210-6217.

14. Fisson, S., et al. 2003. Continuous activation of autoreactive CD4+CD25+ regulatory T cells in the steady state. J. Exp. Med. 198:737-746.

15. Salomon, B., et al. 2000. B7/CD28 costimulation is essential for the homeostasis of the CD4+CD25+ immunoregulatory $\mathrm{T}$ cells that control autoimmune diabetes. Immunity. 12:431-440.

16. Yamazaki, S., et al. 2003. Direct expansion of functional CD25+ CD4+ regulatory $\mathrm{T}$ cells by antigenprocessing dendritic cells. J. Exp. Med. 198:235-247.

17. Kubo, T., et al. 2004. Regulatory T cell suppression and anergy are differentially regulated by proinflammatory cytokines produced by TLR-activated dendritic cells. J. Immunol. 173:7249-7258.

18. Akira, S., and Hemmi, H. 2003. Recognition of pathogen-associated molecular patterns by TLR family. Immunol. Lett. 85:85-95.

19. Caramalho, I., et al. 2003. Regulatory T cells selectively express toll-like receptors and are activated by lipopolysaccharide. J. Exp. Med. 197:403-411.

20. Takahashi, T., et al. 1998. Immunologic self-tolerance maintained by CD $25+\mathrm{CD} 4+$ naturally anergic and suppressive $\mathrm{T}$ cells: induction of autoimmune disease by breaking their anergic/suppressive state. Int. Immunol. 10:1969-1980.

21. Huehn, J., et al. 2004. Developmental stage, phenotype, and migration distinguish naive- and effector/memory-like CD4+ regulatory T cells. J. Exp. Med. 199:303-313.

22. Hori, S., Nomura, T., and Sakaguchi, S. 2003. Control of regulatory $T$ cell development by the transcription factor Foxp3. Science. 299:1057-1061.

23. Fontenot, J.D., Gavin, M.A., and Rudensky, A.Y. 2003. Foxp3 programs the development and function of CD4+CD25+ regulatory T cells. Nat. Immunol. 4:330-336.

24. Khattri, R., Cox, T., Yasayko, S.A., and Ramsdell, F. 2003. An essential role for Scurfin in CD4+CD25+ T regulatory cells. Nat. Immunol. 4:337-342.

25. Yang, Y., Huang, C.T., Huang, X., and Pardoll,
D.M. 2004. Persistent Toll-like receptor signals are required for reversal of regulatory $\mathrm{T}$ cell-mediated CD8 tolerance. Nat. Immunol. 5:508-515.

26. Komai-Koma, M., Jones, L., Ogg, G.S., Xu, D., and Liew, F.Y. 2004. TLR2 is expressed on activated T cells as a costimulatory receptor. Proc. Natl. Acad. Sci. U. S. A. 101:3029-3034.

27. Hirschfeld, M., Ma, Y., Weis, J.H., Vogel, S.N., and Weis, J.J. 2000. Cutting edge: repurification of lipopolysaccharide eliminates signaling through both human and murine toll-like receptor 2. J. Immunol. 165:618-622.

28. Re, F., and Strominger, J.L. 2004. IL-10 released by concomitant TLR2 stimulation blocks the induction of a subset of Th1 cytokines that are specifically induced by TLR 4 or TLR 3 in human dendritic cells. J. Immunol. 173:7548-7555.

29. Netea, M.G., Van der Meer, J.W., and Kullberg, B.J. 2004. Toll-like receptors as an escape mechanism from the host defense. Trends Microbiol. 12:484-488.

30. Hermoso, M.A., Matsuguchi, T., Smoak, K., and Cidlowski, J.A. 2004. Glucocorticoids and tumor necrosis factor alpha cooperatively regulate tolllike receptor 2 gene expression. Mol. Cell. Biol. 24:4743-4756.

31. Peng, G., et al. 2005. Toll-like receptor 8-mediated reversal of CD4+ regulatory T cell function. Science. 309:1380-1384.

32. Belkaid, Y., Piccirillo, C.A., Mendez, S., Shevach, E.M., and Sacks, D.L. 2002. CD4(+)CD25(+) regulatory $\mathrm{T}$ cells control Leishmania major persistence and immunity. Nature. 420:502-507.

33. Netea, M.G., et al. 2002. The role of toll-like receptor (TLR) 2 and TLR4 in the host defense against disseminated candidiasis. J. Infect. Dis. 185:1483-1489.

34. Kirschning, C.J., and Schumann, R.R. 2002. TLR2: cellular sensor for microbial and endogenous molecular patterns. Curr. Top. Microbiol. Immunol. 270:121-144.

35. Takeuchi, O., et al. 1999. Differential roles of TLR2 and TLR4 in recognition of gram-negative and gram-positive bacterial cell wall components. Immunity. 11:443-451.

36. Adachi, O., et al. 1998. Targeted disruption of the MyD88 gene results in loss of IL-1- and IL-18-mediated function. Immunity. 9:143-150. 